\title{
Efficacy and Safety Profile of Ivosidenib in the Management of Patients with Acute Myeloid Leukemia (AML): An Update on the Emerging Evidence
}

\author{
Galia Stemer' \\ Jacob M Rowe ${ }^{2-4}$ \\ Yishai Ofran ${ }^{2,4}$ \\ 'Institute of Hematology, Ha'Emek \\ Medical Center, Afula, Israel; \\ ${ }^{2}$ Department of Hematology and Bone \\ Marrow Transplantation, Rambam Health \\ Care Campus, Haifa, Israel; ${ }^{3}$ Department \\ of Hematology, Shaare Zedek Medical \\ Center, Jerusalem, Israel; ${ }^{4}$ The Ruth and \\ Bruce Rappaport Faculty of Medicine \\ Technion, Haifa, Israel
}

\begin{abstract}
The isocitrate dehydrogenase enzyme, catalyzing isocitrate conversion to $\alpha$-ketoglutarate $(\alpha \mathrm{KG})$ in both the cell cytoplasm and mitochondria, contributes to the production of dihydronicotinamide-adenine dinucleotide phosphate (NADPH) as a reductive potential in various cellular processes. $I D H 1$ gene mutations are revealed in up to $20 \%$ of the patients with acute myeloid leukemia (AML). A mutant IDH enzyme, existing in the cell cytoplasm and possessing neomorphic activity, converts $\alpha \mathrm{KG}$ into oncometabolite R-2-hydroxyglutarate (R-2-HG) that accumulates in high amounts in the cell and inhibits $\alpha \mathrm{KG}$-dependent enzymes, including epigenetic regulators. The resultant alteration in gene expression and blockade of differentiation ultimately lead to leukemia development. Myeloid differentiation capacity can be restored by obstruction of the mutant enzyme, inducing substantial reduction in R-2-HG levels. Ivosidenib, a potent selective inhibitor of mutant $I D H 1$, is a differentiating agent shown to be clinically effective in newly diagnosed AML (ND-AML) and relapsed/refractory (R/R) AML harboring this mutation. The drug is approved by the Food and Drug Administration (FDA) as a single-agent treatment for R/R AML. Significance of mutated IDHI targeting and a potential role of ivosidenib in AML management, when used either as a single agent or as part of combination therapies, will be reviewed herein.
\end{abstract}

Keywords: mutant $I D H 1$, acute myeloid leukemia, ivosidenib

\section{Introduction}

Acute myeloid leukemia (AML) is a heterogeneous hematological malignancy distinguished by a variety of recurring mutated genes. ${ }^{1,2}$ The past decade has witnessed considerable advances in unraveling molecular, genetic, and epigenetic underpinnings of AML and in the identification of its new diagnostic and prognostic markers.

In the past years, new drugs, including a wide range of small-molecule inhibitors, have been developed and approved by the Food and Drug Administration (FDA), thus extending the therapeutic landscape for AML. ${ }^{3}$

Recurrent mutations in isocitrate dehydrogenase 1 and 2 (IDH1 and IDH2) are among most prevalent in AML (found in about $20 \%$ of the patients). ${ }^{4}$

The breakthrough in the identification of targetable genetic aberrations in AML, has provided a unique platform for the development of targeted therapeutic agents
Correspondence: Jacob M Rowe Department of Hematology, Shaare

Zedek Medical Center, Shmu'el Bait St 12 Jerusalem, 9103I, Israel

Tel +972-2-6555204

Fax +972-2-6555755

Email rowe@rambam.health.gov.il 
for the management of this aggressive disease. Ivosidenib, a potent selective inhibitor of the $I D H 1$ mutant protein has been currently approved in the United States (US) for the treatment of adult AML patients harboring this mutation who are relapsed or refractory $(\mathrm{R} / \mathrm{R})$ to prior therapy and individuals $\geq 75$ years of age or those with comorbidities, precluding the use of intensive induction chemotherapy. ${ }^{5}$

This review will focus on IDH1 mutations and discuss the mechanism of action of ivosidenib, as well as its role in AML treatment.

\section{The Role of IDHI Mutation in Leukemogenesis}

Isocitrate dehydrogenase 1 (IDH1) is one of the key enzymes that plays a role in cellular metabolism. It catalyzes the reversible oxidative decarboxylation of isocitrate to alpha ketoglutarate $(\alpha \mathrm{KG})$ in the cell cytoplasm, with simultaneous reduction of $\mathrm{NADP}^{+}$to $\mathrm{NADPH} .^{2}$

Alteration in cellular metabolism is one of the key characteristics of cancer cells. This phenomenon is known as the "Warburg effect", first described in $1924{ }^{6}$ Cancer cells, including those of AML, preferentially utilize glucose to generate energy, ie, use the tricarboxylic acid (TCA) cycle $^{6}$ even in the presence of oxygen. ${ }^{7}$

A loss-of-function mutation in IDH1 shifts the products of the TCA cycle, such as $\alpha \mathrm{KG}$, towards the production of 2-hydroxyglutarate $(2 \mathrm{HG}) .{ }^{8}$ In AML cells that harbor an $I D H 1$ mutation a significant elevation in $2 \mathrm{HG}$ levels is seen. The most common IDH1 mutation recurrently occurring in glioma and leukemia is the one that leads to the replacement of arginine located at position 132 of the IDH1 gene by histidine. ${ }^{9}$ Mutations at position 132 $\left(I D H 1^{R 132}\right)$ are the most prevalent recurrent $I D H 1$ mutations. The mutated IDH is capable of cell transformation through alteration of the activity of 2HG-dependent enzymes. ${ }^{5}$ The mutated IDH preferably produces the (R) $2 \mathrm{HG}$ enantiomer. ${ }^{10}$ The (R) enantiomer accumulation leads to differentiation arrest and leukemia development. Fortunately, and fundamental to the therapeutic potential of IDH inhibition, this effect on cell differentiation is reversible when levels of (R) $2 \mathrm{HG}$ are restored to normal. ${ }^{10,11}$ Additionally, a decrease in $\alpha \mathrm{KG}$ levels is accompanied with an increase in $2 \mathrm{HG}$ levels. Consequently, 2HG acts as a competitive inhibitor of $\alpha$ KG-dependent reactions. The resultant hypermethylation of DNA and histones leads to the differentiation blockade ultimately promoting leukemogenesis. ${ }^{5}$
These findings have given rise to a hypothesis that inhibition of IDH1 activity in $I D H 1^{R 132}$ cases could reverse this abnormal accumulation and induce differentiation of leukemic cells. $^{12}$

\section{Prognostic Significance of IDHI RI 32 Mutation}

$I D H 1^{R 132}$ was first determined to be a recurring oncogenic mutation in glioblastoma. Parsons et al, exploring the genomic landscape of glioblastoma multiforme (GBM), have identified mono-allelic, missense, point mutations in $I D H 1$ as the most frequent aberration in this disease. ${ }^{13}$ $I D H 1^{R 132}$ is reported to be present in $>80 \%$ of the adult patients with secondary GBMs and in over $70 \%$ of the adults with grade 2 and grade 3 gliomas. ${ }^{14}$ Notably, IDH mutations have also been observed in over $50 \%$ of chondrosarcoma cases as well as in up to $20 \%$ of cholangiocarcinomas and in rare cases of paraganglioma, colon, prostate, and lung cancers. ${ }^{14}$

The prognostic significance of $I D H-1$ mutations varies among different cancer types. For instance, in GBM, $I D H$ mutations are associated with a longer overall survival (OS), amounting to 31 months, relative to only 15 months in patients with $I D H$ wild-type. ${ }^{15}$ While being frequent among patients with cholangiocarcinoma, the IDHI mutation has not been found to affect their OS. ${ }^{16}$

In AML, the $I D H 1^{R 132}$ mutation is revealed as a recurring event ${ }^{17}$ in $6-10 \%$ of the patients. ${ }^{18}$ Notably, among AML patients presenting with normal cytogenetics, the incidence of $I D H I$ mutation has been reported to reach $16 \%{ }^{4,19} \mathrm{IDH} 1$ mutations are known to frequently coexist with other molecular aberrations, such as nucleophosmin 1 (NPM1) mutations and partial tandem duplication of mixed lineage leukemia gene $(M L L-P T D) .{ }^{12,20}$ Mutations in the IDH1 gene are also found, albeit at a lower rate, in other myeloid malignancies, equating to up to $12 \%$ and $8 \%$ among patients with myelodysplastic syndrome (MDS) and myeloproliferative neoplasms (MPNs), respectively, ${ }^{10,21,22}$ and their significant presence changes among the different hematologic malignancies. This will be discussed in detail herein.

IDH1 mutations are considered driver mutations that play a role in leukemogenesis and are observed in preleukemic hematopoietic stem cells (HSCs) and progenitors. ${ }^{21}$ Notably, acquisition of an IDH1 mutation may promote transformation of MDS and MPN to AML. ${ }^{21,23,24}$ 


\section{IDHI Mutations in MDS}

A recent analysis of genetic abnormalities reported $I D H I$ mutations in about $3 \%{ }^{25}$ of the samples obtained from 944 MDS patients. These mutations often co-existed with SRSF2 and DNMT3A mutations, while being mutually exclusive with TET2 mutations. ${ }^{25}$

Molenaar et al, evaluating the incidence of IDH1 mutations in 868 low-risk and 536 high-risk patients with MDS, ${ }^{15}$ reported increased frequency of these aberrations in high-risk compared to low-risk patients. The presence of $I D H 1$ mutations appeared to be associated with shorter survival, particularly in individuals with low-risk disease. ${ }^{26,27}$ Moreover, high-risk MDS patients harboring mutated $I D H 1^{R 132}$ were reported to be more prone to AML transformation. ${ }^{28}$ Notably, in such cases, typical AML mutations such as FLT3, PTPN11, WT1, NPM1, were also present. ${ }^{28}$ In a recent study from the MD Anderson Cancer Center, $1.6 \%$ $(\mathrm{n}=17)$ out of 1042 MDS patients displayed the $I D H 1^{R 132}$ mutation $^{2}$ and demonstrated unique clinical and pathologic features, including elevated absolute neutrophil counts, a higher percentage of bone marrow blasts, and a trend towards increased platelet counts relative to those observed in MDS patients with normal $I D H l^{2}{ }^{2}$ Overall, all these studies point to an association between $I D H$ mutations and a more advanced disease stage.

Over the last decade, several studies assessed the prognostic impact of $I D H 1$ mutations in MDS. A study from the Mayo Clinic, evaluating a cohort of 277 MDS patients, identified $I D H$ mutations in $12 \%$ of the patients, $2.6 \%$ of whom displayed IDHI alterations (mostly R132-S). ${ }^{29}$ While the frequency of $I D H 1$ mutations varied between MDS subtypes, all but one patient in that study had a normal karyotype. ${ }^{29}$ Importantly, in a multivariate analysis, these mutations emerged as the only factor associated with reduced leukemia-free survival. ${ }^{29}$ A later study, incorporating 97 patients with MDS, confirmed an association of IDHI mutations with shorter OS and progression-free survival (PFS) ${ }^{30}$

In summary, the incidence of $I D H 1$ mutations in MDS is lower than that reported in AML and appears to increase with a rising MDS risk score, implying involvement of these mutations in disease progression. ${ }^{27}$

The mechanisms underlying MDS transformation to secondary AML (sAML) can be explained by one of the following two models ${ }^{2,27}$ :

(a) A linear model, using results of bulk sequencing analysis, suggests accumulation of serial mutations during disease development from non-mutated HSCs to clonal hematopoiesis, MDS and ultimately to sAML.

(b) A nonlinear model of clonal evolution suggests that mutation accumulation in various stem cell compartments results in vast subclonal diversity in MDS stem cells. While some of such subclones induce MDS, others first function as pre-AML and later as AML stem cells.

In both situations, the low prevalence of IDH1 mutation in MDS is in favor of its strong leukemogenesis potential.

\section{IDHI Mutations in MPN}

The World Health Organization (WHO) classifies the following types of myeloproliferative neoplasms (MPNs) related to $J A K 2, C A L R$, and $M P L$ gene mutations: polycythemia vera (PV), essential thrombocytopenia (ET), primary myelofibrosis (PMF) and prefibrotic PMF (pre-PMF). ${ }^{31}$ In general, IDH1 mutations are rare in chronic-phase MPNs and have been reported in $0 \%$ of PV and ET patients and only in $1 \%$ of PMF patients. ${ }^{32}$ Several studies demonstrated that the frequency of $I D H 1$ mutations in MPN increased during the blast phase. Green et al evaluating 16 patients with blast/leukemic phase of pre-existing JAK2-mutated MPN, identified IDH mutations in five of these patients, three of whom harbored $I D H I^{R I 32} \cdot{ }^{17}$ In a study from the Mayo Clinic, IDH mutations were detected in 9 (4\%) of the 227 patients with either chronic or blast-phase MPN, screened for these aberrations. Of note, five of the nine individuals displayed $I D H 1^{R 132}$ $(2.2 \%) .{ }^{33}$ The cumulative $I D H(1+2)$ mutational frequency was found to be about $4 \%$ for patients in the chronic phase of the disease and $21 \%$ for those with blast-phase MPN. ${ }^{33}$

$I D H 1^{R 132}$ has been recently established as one of the mutations associated with rapid progression to myelofibrosis $^{34}$ and AML transformation. ${ }^{24}$

The co-occurrence of mutated IDHI with other mutations, specifically SRSF2, may accelerate progression to AML and shorten leukemia-free survival. ${ }^{35}$ This is particularly true of PMFs with mutated SRSF2-, where $13 \%$ of the patients have been reported to display $I D H 1$ mutations relative to only $1 \%$ observed in $S R S F 2$ wild-type PMFs. ${ }^{35}$ Likewise, PMF patients presenting with concurrent JAK2 and $I D H 1^{R 132}$ mutations are suggested to be at risk of disease progression. ${ }^{36}$

A recent analysis from the MD Anderson Cancer Center, evaluating the role of IDH $1 / 2$ inhibitors in the management of post-MPN AML, included five patients with $I D H-1$ mutation who were treated with ivosidenib either as a single agent or as part of a combination 
regimen. ${ }^{37}$ Although none of the patients receiving ivosidenib monotherapy achieved complete remission, they demonstrated a significant reduction in the percentage of blasts along with a clinical benefit of over 6 months.

In summary, IDH1 ${ }^{R 132}$ mutation has been associated with poor prognosis in both MDS and MPN. The incidence of $I D H 1^{R 132}$ is increased in late-stage MPN and high-risk MDS (20\%) compared to early-stage and lowrisk disease $(<4 \%) . I D H 1^{R 132}$ is frequently present in secondary AML, and is thus considered as one of the factors contributing to the evolution of chronic MDS and MPN to full-blown leukemia. ${ }^{29,36}$

\section{IDHI Mutations in AML}

IDH1 mutations are recognized as one of the most common genetic abnormalities in AML.

In a study conducted at the Washington University, $\mathrm{IDH} 1$ mutations emerged as recurring genetic alterations in 16 of 188 AML patient samples $(8.5 \%) .{ }^{4}$ This finding was further confirmed by Paschka et al in $14 \%$ of the AML patients included in that study. ${ }^{38}$ In a retrospective mutational analysis from the Memorial Sloan Kettering Cancer Center, including 398 AML patient samples from E1900 study [by the Eastern Cooperative Oncology Group (ECOG)] and 104 validation cohort samples, somatic alterations were found in $97.3 \%$ of the samples, with IDH1 mutation revealed in $7 \%$ of these cases. ${ }^{39}$ Notably, among patients with intermediate-risk normal cytogenetics, this prevalence could reach $15-20 \%{ }^{40}$ It was also found, in the ECOG-AML cohort, that IDH1 (and IDH2) mutations were mutually exclusive. Expression of IDH mutants disrupted TET2 catalytic function in cells and impaired hematopoietic differentiation causing facilitation of stem/progenitor cell marker expression, suggesting a leukemogenic effect. ${ }^{41}$

The prognostic significance of $I D H I$ mutations in AML is not fully elucidated. Similar to other genetic interactions in AML, there seems to be a difference in the prognosis depending on whether the IDHI mutation appears in isolation or in combination with other mutations. In the Medical Research Council (MRC) 10 and 12 studies, among 1333 young adult patients with AML, $I D H 1^{R 132}$ was found to be associated with high relapse rates. ${ }^{42}$ At the same time, another large study including 826 AML patients reported comparable OS for $I D H-W T$ and $I D H$-mutated AMLs. ${ }^{43}$ In the aforementioned ECOG E1900 study, patients with intermediate-risk AML harboring both NPMI and IDHI mutations demonstrated a superior 3-year OS relative to that observed in patients with mutant NPM1 and wild-type IDH1 ( $89 \%$ vs $31 \%$, $\mathrm{P}<0.001) .{ }^{39}$ A recent meta-analysis incorporating data of 33 reported studies ${ }^{44}$ concluded that in AML, the presence of mutant $I D H 1$ was associated with reduced OS (HR, 1.17; $\mathrm{P}=0.0047$ ) and event-free survival (EFS; HR 1.29; $\mathrm{P}=0.0110)$ compared to those found in patients with wildtype $I D H 1$, particularly in normal-karyotype AML (CNAML). Likewise, IDH1 single-nucleotide-polymorphism (SNP) rs11554137 appeared to correlate with an inferior OS (HR 1.34; $\mathrm{P}=0.0294$ ).

Given the genetic heterogeneity of AML, Dunlap et al made an attempt to assess the clinical impact of coexisting mutations on the outcome of forty patients (median age 60 years) with normal cytogenetics and mutated NPM1 who were FLT3-ITD negative. ${ }^{45}$ The 5 -year OS and diseasefree survival (DFS) of the study group were $54.8 \%$ and $42.8 \%$, respectively. The presence of triple mutations in $N P M I+D N M T 3 a$ and IDHI was associated with a trend towards reduced OS, irrespective of potential confounders such as age and $\mathrm{WBC}$ at presentation. ${ }^{45}$

\section{Ivosidenib Mechanism of Action}

Ivosidenib (known as AG-120 and AGI-16,678) is a highly specific, allosteric, reversible inhibitor of mutated IDH1 (Tibsovo, Agios Pharmaceuticals). According to the criteria of the FDA Biopharmaceutical Classification System (BCS) it is defined as a Class II compound (low solubility, high permeability, is mainly metabolized by CYP3A4, and inducing CYP3A enzyme activity).

In a chondrosarcoma cell line model, harboring an endogenous $I D H 1$ mutation was demonstrated to result in a 100-fold increase of both intracellular and extracellular D-2-HG levels, compared to IDH1 wild-type cell lines. Specific inhibition of mutant $I D H 1$ with AGI-5198 led to a $>90 \%$ reduction of D-2-HG levels in a dose-dependent manner and a moderate decrease in the viability of mutant IDH1 cell lines. However, this did not significantly affect the tumorigenic properties of these cell lines, which precluded the use of this compound in clinical studies. ${ }^{46}$ Ivosidenib was the first IDH1 enzyme inhibitor that demonstrated a proof of concept in clinical trials. A US multicenter Phase 1 study, including 168 patients with various types of $I D H 1$-mutant solid tumors who received at least one dose of ivosidenib with dose escalation (range $100 \mathrm{mg}-1200 \mathrm{mg}$ ), demonstrated $98 \%$ inhibition of plasma 2-HG in patients with chondrosarcoma and cholangiocarcinoma after continuous ivosidenib treatment for 1 week. 
The resultant 2-HG levels appeared to be comparable to those observed in healthy subjects and persisted throughout the treatment period. ${ }^{47}$ Likewise, in patients with glioma, mean post-treatment plasma 2-HG levels remained within the normal range. ${ }^{47}$

To determine the effect of mutant IDHI inhibition in primary human AML blast cells, Popovici-Muller et al investigated mutant $I D H 1-R^{132 H}$, mutant IDH1-R ${ }^{132 C}$, and $I D H 1$ wild type in the bone marrow and peripheral blood samples derived from patients that were treated with ivosidenib in an ex vivo assay. In mutant IDHI samples, ivosidenib was found to decrease the level of intracellular 2-HG by $96 \%$ at the lowest administered dose of $0.5 \mu \mathrm{M}$ and by $98.6 \%$ and $99.7 \%$, respectively, when 1 and $5 \mu \mathrm{M}$ doses were used. Ivosidenib induced differentiation of primary mutant $I D H 1-R^{132 H}$ and mutant IDH1-R $R^{132 C}$ blast cells obtained from AML patients treated ex vivo. This was evidenced by enhanced ability of the cells to form differentiated colonies, as well as elevation in the expression of cell-surface differentiation markers and a rise in the proportion of mature myeloid cells. ${ }^{12}$

\section{Pharmacokinetics}

Ivosidenib is rapidly absorbed, reaching a steady state at 14 days and a protein-bound range of $92-96 \%$. High-fat meals may interfere with its absorption and should not precede drug swallowing. ${ }^{48}$ The drug is metabolized in the liver by CYP3A4, being mainly excreted in the feces $(77 \%$ unchanged) and in the urine (10\% unchanged and $7 \%$ metabolized). Concomitant use with moderate/strong CYP3A4 inhibitors, especially antifungal azoles, reduces ivosidenib clearance. ${ }^{49}$ Co-administration of strong CYP3A4 inducers is not recommended and if strongly indicated, a dose reduction of ivosidenib from 500 to $250 \mathrm{mg} /$ day is advised. No dose adjustments of ivosidenib are needed in concomitant use of weak CYP3A4 inhibitor or inducers. ${ }^{48,49}$ Concomitant application of ivosidenib and CYP substrates with narrow therapeutic windows (eg, warfarin, phenytoin) is contraindicated. ${ }^{49}$ Given that ivosidenib is a P-glycoprotein inhibitor, patients should avoid concurrent use of P-glycoprotein substrates (such as verapamil or cyclosporine) while on treatment with ivosidenib.

\section{Ivosidenib for Relapsed AML with IDHI Mutation}

On July 20, 2018, the FDA approved ivosidenib (TIBSOVO, Agios Pharmaceuticals, Inc.) for the use in AML patients harboring an IDHI mutation, while in relapse. The approval was granted based on the findings of a non-randomized, open-label, single-arm, multicenter, phase 1 , dose-escalation and dose-expansion study of ivosidenib prescribed as monotherapy. ${ }^{50}$ Included in that study were patients who could not receive conventional therapy, so the label was limited to patients older than 75 years or those who had comorbidities precluding the use of intensive induction chemotherapy. ${ }^{5}$

Two hundred and fifty-eight patients received ivosidenib in that trial. The median age of enrolled patients was 68 years, 39\% of them were diagnosed with secondary AML, and adverse cytogenetics was reported in $31 \%$. The maximum prescribed ivosidenib dose was $1200 \mathrm{mg}$ per day, while the maximum tolerable dose was not reached. However, no clinical benefit associated with the dose increase was observed and $500 \mathrm{mg}$ was identified as the optimal daily dose. ${ }^{50}$

The efficacy of ivosidenib was assessed based on complete remission (CR) + CR with partial hematologic recovery $(\mathrm{CRh})$ rate, duration of $\mathrm{CR}+\mathrm{CRh}$, as well as conversion of transfusion dependence to transfusion independence. At a median follow-up of 8.3 months, in 174 adult patients with $I D H 1$-mutated R/R AML, the $\mathrm{CR}+$ $\mathrm{CRh}$ rate was 33\% [95\% confidence interval (CI), 26-40] with median response duration of 8.2 (95\% CI, 5.6-12) months, and transfusion independence acquired in $37 \%$ of the patients. ${ }^{51}$

Although the results show a short-term benefit in patients with an unmet medical need, responses to ivosidenib may appear only after several months, as was reported by DiNardo et al in a cohort of $125 \mathrm{R} / \mathrm{R}$ AML patients, treated with ivosidenib. ${ }^{52}$ Responses developed within up to 8 months, with a median response duration of more than 6 months. The CR rate was $22 \%$ with a median duration of over 9 months. Median OS for the total cohort was 9 months, while it equated to 18 months for those who achieved CR. ${ }^{52}$

The most frequent $(20 \%)$ adverse reactions of any grade recorded in patients receiving ivosidenib included diarrhea, febrile neutropenia, leukocytosis, fatigue, nausea, dyspnea, prolongation of the QT interval, edema, anemia, pyrexia, and cough. ${ }^{50}$ Among serious adverse reactions, observed in $5 \%$ of the cases, were QTc interval prolongation $(7.8 \%)$, leukocytosis $(10 \%)$ and differentiation syndrome $(3.9 \%)$.

Concomitant use of ivosidenib and other drugs with the potential of prolonging the QTc interval should be avoided, and these drugs should be replaced with 
alternative treatments if possible. Otherwise, the subjects receiving such drugs should be adequately monitored with electrocardiogram (ECG) control and measurement of serum electrolyte levels, particularly those of potassium and magnesium.

In case the QTc interval is $>480 \mathrm{~ms}$ (grade 2) or $>500$ ms (grade 3), treatment with ivosidenib should be discontinued and restarted after the values return to $\leq 480 \mathrm{~ms}$, with the recommended drug doses of $500 \mathrm{mg} /$ day and $250 \mathrm{mg} /$ day for grade 2 and grade 3 events, respectively. In addition, ECG should be monitored at least once weekly for 2 weeks following resolution. The main drugs that could prolong the QTc interval are fluoroquinolones (eg, ciprofloxacin, moxifloxacin), 5-HT3 antagonists (eg, granisetron, ondansetron), and azole antifungals (eg, fluconazole, voriconazole, posaconazole), which are also strong or moderate CYP3A4 inhibitors and may increase ivosidenib plasma concentrations (worsening potential QTc interval prolongation).

In patients for whom antifungal therapy is necessary, alternative treatments should be considered like aerosolized liposomal amphotericin B, added to systemic antifungal treatment, despite limited data, ${ }^{53,54}$ intravenous agents such as echinocandins (caspofungin, micafungin, and anidulafungin) or low-dose amphotericin B. Another option may be the use of isavuconazole as an oral agent with a spectrum of activity similar to that of posaconazole or voriconazole, which are moderate CY34A inhibitors, without QTc prolongation effects. ${ }^{49}$

As mentioned above, differentiation syndrome (DS) was identified as another serious adverse effect ${ }^{50}$ in patients treated with ivosidenib, who experienced the symptoms, similar to those observed in patients receiving all-trans retinoic acid (ATRA) for acute promyelocytic leukemia (APL). These reactions were apparently caused by cytokine release from differentiating myeloid blasts. ${ }^{55,56}$ DS was reported to evolve late during therapy, ${ }^{57}$ with median onset time of 29 days (range 5-59 days) and with $37 \%$ of the patients developing leukocytosis. There were also reports of patients presenting with treatment-related myopericarditis and cardiogenic shock. $^{57}$

The recommended DS treatment included use of steroids, diuretics with or without hydroxyurea, and non/invasive ventilation. These measures led to the resolution of symptoms in almost $90 \%$ of the cases. Of note, none of the patients experienced grade 4 or lethal events. Treatment with ivosidenib that has been withheld in some patients, could be safely renewed at the standard daily dose of $500 \mathrm{mg} .{ }^{21,50,57,58}$ Yet, caution is advised since there is no agreement regarding the optimal timing and dosage for ivosidenib re-introduction after DS.

A recent systematic analysis of $\mathrm{DS}$ in $\mathrm{R} / \mathrm{R}$ AML patients treated with ivosidenib (NCT02074839) or the IDH2 inhibitor, enasidenib (NCT01915498), conducted by the FDA $^{58}$ estimated potential DS risk associated with ivosidenib therapy in a multivariable model. The assessed parameters included baseline bone marrow and peripheral blood blast percentages, secondary versus de novo AML, WBC count, and LDH levels. Other parameters evaluated in the multivariate analysis were prior hematopoietic stem cell transplantation (HSCT) and the presence of TET2 mutations. Peripheral blast count $\geq 25 \%$ and bone marrow blast count $\geq 48 \%$ along with a median WBC count above $10 \times 10^{3}, \mathrm{LDH}$ above the upper limit of normal, prior HSCT and the presence of TET2 mutations were associated with higher relative risk of DS. Potential DS risk was suspected when two or more criteria were found to be positive within 7 days of appearance. DS was considered moderate when fulfilled two or three criteria and severe when fulfilled four or more criteria. ${ }^{51,58,59}$ It is noteworthy that morphological evidence of cellular differentiation in blood or bone marrow was not considered a requirement for potential DS.

Despite the imminent potential for the development of DS with possible fatal complications, the reported data from different studies suggest that this phenomenon is infrequent, occurs late during exposure to ivosidenib and the assumption of a favorable outcome with prompt early treatment is fair. ${ }^{50,56,57,60,61}$

\section{Ivosidenib as First-Line Therapy for AML with Mutant IDHI}

On May 2, 2019, the FDA approved ivosidenib (TIBSOVO, Agios Pharmaceuticals, Inc.) for the application in newly-diagnosed AML with a susceptible $I D H I$ mutation, in patients $\geq 75$ years of age or those with comorbidities precluding the use of intensive induction chemotherapy.

The approval was granted based on the data from an open-label, single-arm, multicenter clinical trial (Study AG120-C-001, NCT02074839) of single-agent ivosidenib used for newly diagnosed AML with an IDH1 mutation. ${ }^{60}$ Patients aged $\geq 75$ years or those who met at least one of the following criteria were enrolled in the study: baseline 
ECOG performance status (ECOG PS) $\geq 2$, severe cardiac or pulmonary disease, hepatic impairment with bilirubin $>1.5$ times the upper limit of normal, or creatinine clearance $<45 \mathrm{~mL} / \mathrm{min}$. Twenty-eight patients were treated (median age 77 years; range: 64-87 years); 22 (79\%) of them had therapy-associated AML or AML with myelodysplasia-related alterations. Ivosidenib was given orally at a daily dose of $500 \mathrm{mg}$ and was discontinued due to disease progression, unacceptable toxicity, or HSCT. CR $+\mathrm{CRh}$ was achieved in $12 / 28$ patients (42.9\%); 7/17 $(41.2 \%)$ transfusion-dependent patients acquired transfusion independence that maintained for a minimum of 8 weeks. Two of the 28 patients received HSCT. Based on the results of that study, the FDA recommended an oral once-daily ivosidenib dose of $500 \mathrm{mg}$ (with or without food) that could be administered until AML progression or unacceptable toxicity. To ensure clinical response in patients who did not experience disease progression or unacceptable toxicity, the therapy duration of a minimum of 6 months was recommended. ${ }^{62}$

Adverse events, recorded in at least $25 \%$ of the study participants, included the following: nausea, diarrhea, decreased appetite, fatigue, edema, leukocytosis, arthralgia, abdominal pain, dyspnea, DS and myalgia. Prescribing information contained a warning addressed to both healthcare practitioners and patients regarding the risk of DS that could be life-threatening or fatal.

A recently conducted multicenter, open-label, phase 1 study assessed safety and tolerability of induction and consolidation combination regimens, including ivosidenib (or enasidenib) and intensive chemotherapy, in ND-AML patients with mutant IDH1 or IDH2 ${ }^{61}$ The study also analyzed clinical responses. Next-generation sequencing (NGS) was used to evaluate the mutational profile at the starting point of treatment with ivosidenib as well as at the time of the best response. Responses were assessed by multi-parameter flow cytometry and digital polymerase chain reaction (PCR) assay to evaluate measurable minimal residual disease (MRD). Despite the fact that only a small number of patients were assessed by both methods, the promising findings of $I D H 1$ clearance in $39 \%$ of the patients (16/41) and MRD achievement in $80 \%$ of them $(16 / 20)$ could make ivosidenib combined with intensive chemotherapy an attractive treatment option for newly diagnosed AML patients with mutated IDH1. ${ }^{61}$

Prospective studies are still needed to identify the optimal method for the assessment of mutant IDHI clearance that would provide the most accurate information, especially in clinical practice.

A number of ongoing clinical trials are evaluating potential expansion of the indications for the use of ivosidenib in IDH1-mutated AML and MDS (Table 1).

In contrast to the FDA, ivosidenib has not yet been approved in Europe. The European Medicines Agency (EMA) had reservations about the data, partly related to efficacy and lack of better control, leading the sponsor in November 2020 to withdraw the application. The lack of approval in Europe relates to both relapsed and newly diagnosed AML patients.

\section{Resistance to Ivosidenib}

Several studies have addressed the issue of resistance mechanisms to ivosidenib. Based on patient response, the following two types of drug resistance are defined: primary resistance refers to the lack of response to initial therapy; secondary resistance refers to the development of drug resistance following initial response to treatment.

Data from a recently published international phase 1 study, exploring molecular mechanisms underlying primary and secondary resistance to ivosidenib in $R / R$ AML with mutant $I D H 1^{63}$ demonstrate an association of mutations in receptor tyrosine kinase (RTK) pathway genes with both primary and secondary resistance in this patient population. Notably, KRAS mutations appear to be more frequent at relapse/progression than at baseline. ${ }^{63}$

These findings are in line with previously reported evidence of an association between baseline mutations in RTK pathway genes and primary resistance to ivosidenib. $^{50}$ The biological processes explaining a causal link between RTK pathway mutations and both primary and secondary resistance to ivosidenib remain to be elucidated.

According to one hypothesis, proliferative and prosurvival impacts of RTK pathway stimulation could be potent oncogenic signals sufficient to diminish 2-HG dependence. Another hypothesis suggests involvement of RTK pathway-activating mutations in differentiation inhibition, which remains enforced after the start of ivosidenib therapy. It is also hypothesized that $I D H 1 / 2$ mutations could contribute to the activation of certain components in RTK signaling, that might be irreversible in response to ivosidenib in patients with co-existing RTK pathway mutations. $^{63}$

A mechanism mediating secondary resistance could be attributed to the restoration of $2-\mathrm{HG}$ caused by mutations 
Table I Current Clinical Trials in AML and MDS

\begin{tabular}{|c|c|c|c|c|c|}
\hline $\begin{array}{l}\text { NCT } \\
\text { Number }\end{array}$ & Title & Conditions & Interventions & $\begin{array}{l}\text { Estimated } \\
\text { Patient } \\
\text { Enrollment }\end{array}$ & Characteristics \\
\hline NCT04493I64 & $\begin{array}{l}\text { CPX-35I and ivosidenib for } \\
\text { the treatment of IDHI } \\
\text { mutated acute myeloid } \\
\text { leukemia or high-risk } \\
\text { myelodysplastic syndrome }\end{array}$ & $\begin{array}{l}\text { - Acute myeloid leukemia } \\
\text { with gene mutations } \\
\text { - Myelodysplastic syndrome } \\
\text { - Myeloproliferative neoplasm } \\
\text { - Recurrent/Refractory acute } \\
\text { myeloid leukemia }\end{array}$ & $\begin{array}{l}\text { - Drug: Ivosidenib } \\
\text { - Drug: } \\
\text { Liposome-encapsulated } \\
\text { daunorubicin- } \\
\text { cytarabine }\end{array}$ & 30 & Phase 2 \\
\hline NCT0425005I & $\begin{array}{l}\text { Ivosidenib and combination } \\
\text { chemotherapy for the } \\
\text { treatment of IDHI mutant } \\
\text { relapsed or refractory acute } \\
\text { myeloid leukemia }\end{array}$ & $\begin{array}{l}\text { - Recurrent/Refractory acute } \\
\text { myeloid leukemia } \\
\text { - Recurrent/Refractory } \\
\text { myelodysplastic syndrome } \\
\text { - Recurrent } \\
\text { Myeloproliferative neoplasm }\end{array}$ & $\begin{array}{l}\text { - Drug: Cytarabine } \\
\text { - Biological: Filgrastim } \\
\text { - Drug: Fludarabine } \\
\text { - Drug: Fludarabine } \\
\text { Phosphate } \\
\text { - Drug: Ivosidenib }\end{array}$ & 25 & Phase I \\
\hline NCT04I76393 & $\begin{array}{l}\text { A China bridging study of } \\
\text { ivosidenib in } r / r \text { AML subjects } \\
\text { with an IDHI mutation }\end{array}$ & $\begin{array}{l}\text { - Relapsed/Refractory acute } \\
\text { myeloid leukemia }\end{array}$ & - Drug: Ivosidenib & 30 & Phase I \\
\hline NCT0383977I & $\begin{array}{l}\text { A study of ivosidenib or } \\
\text { enasidenib in combination with } \\
\text { induction therapy and } \\
\text { consolidation therapy, } \\
\text { followed by maintenance } \\
\text { therapy in patients with newly } \\
\text { diagnosed acute myeloid } \\
\text { leukemia or myedysplastic } \\
\text { syndrome EB2, with an IDHI } \\
\text { or IDH2 mutation, } \\
\text { respectively, eligible for } \\
\text { intensive chemotherapy }\end{array}$ & $\begin{array}{l}\text { - Acute myeloid leukemia } \\
\text { - Myelodysplastic syndrome } \\
\text { with excess blasts-2 }\end{array}$ & $\begin{array}{l}\text { - Drug: AG-120 } \\
\text { - Drug: Placebo or AG- } \\
\text { I20 } \\
\text { - Drug: AG-22I } \\
\text { - Drug: Placebo for AG- } \\
221\end{array}$ & 968 & Phase 3 \\
\hline NCT03503409 & $\begin{array}{l}\text { IDHI (AG I20) inhibitor in } \\
\text { patients with IDHI-mutated } \\
\text { myelodysplastic syndrome }\end{array}$ & $\begin{array}{l}\text { - Myelodysplastic syndromes } \\
\text { - Acute myeloid leukemia }\end{array}$ & - Drug: AG-120 & 68 & Phase 2 \\
\hline NCT0347I260 & $\begin{array}{l}\text { Ivosidenib and venetoclax with } \\
\text { or without azacitidine in } \\
\text { treating participants with } \\
\text { IDHI-mutated hematologic } \\
\text { malignancies }\end{array}$ & $\begin{array}{l}\text { - High-risk myelodysplastic } \\
\text { syndrome } \\
\text { - IDHI.RI32X } \\
\text { - Myeloproliferative neoplasm } \\
\text { - Recurrent/Refractory acute } \\
\text { myeloid leukemia }\end{array}$ & $\begin{array}{l}\text { - Drug: Azacitidine } \\
\text { - Drug: Ivosidenib } \\
\text { - Other: } \\
\text { Pharmacokinetic Study } \\
\text { - Drug: Venetoclax }\end{array}$ & 48 & Phase $1 / 2$ \\
\hline NCT03245424 & $\begin{array}{l}\text { Ivosidenib expanded access } \\
\text { program in relapsed/refractory } \\
\text { aml with an IDHI mutation }\end{array}$ & $\begin{array}{l}\text { - Acute myeloid leukemia } \\
\text { - Relapsed adult AML } \\
\text { - Relapsed pediatric AML }\end{array}$ & $\begin{array}{l}\text { - Drug: Ivosidenib (AG- } \\
\text { 120) }\end{array}$ & & \\
\hline NCT03I73248 & $\begin{array}{l}\text { Study of AG-I } 20 \text { (ivosidenib) } \\
\text { vs placebo in combination with } \\
\text { azacitidine in patients with } \\
\text { previously untreated acute } \\
\text { myeloid leukemia with an } \\
\text { IDHI mutation }\end{array}$ & $\begin{array}{l}\text { - Newly diagnosed acute } \\
\text { myeloid leukemia } \\
\text { - Untreated AML } \\
\text { - AML arising from } \\
\text { myelodysplastic syndrome }\end{array}$ & $\begin{array}{l}\text { - Drug: AG-120 } \\
\text { (ivosidenib) with } \\
\text { azacitidine } \\
\text { - Drug: Placebo with } \\
\text { azacitidine }\end{array}$ & 200 & Phase 3 \\
\hline
\end{tabular}

(Continued) 
Table I (Continued).

\begin{tabular}{|c|c|c|c|c|c|}
\hline $\begin{array}{l}\text { NCT } \\
\text { Number }\end{array}$ & Title & Conditions & Interventions & $\begin{array}{l}\text { Estimated } \\
\text { Patient } \\
\text { Enrollment }\end{array}$ & Characteristics \\
\hline NCT02677922 & $\begin{array}{l}\text { A safety and efficacy study of } \\
\text { oral AG-I } 20 \text { plus } \\
\text { subcutaneous azacitidine and } \\
\text { oral AG-22I plus } \\
\text { subcutaneous azacitidine in } \\
\text { subjects with newly diagnosed } \\
\text { acute myeloid leukemia (AML) }\end{array}$ & - Leukemia, myeloid, acute & $\begin{array}{l}\text { - Drug: AG-120 } \\
\text { - Drug: Azacitidine } \\
\text { - Drug: AG-22I }\end{array}$ & $|3| *$ & Phase $1 / 2$ \\
\hline NCT02632708 & $\begin{array}{l}\text { Safety study of AG-I } 20 \text { or AG- } \\
22 I \text { in combination with } \\
\text { induction and consolidation } \\
\text { therapy in participants with } \\
\text { newly diagnosed acute myeloid } \\
\text { leukemia (AML) with an IDHI } \\
\text { and/or IDH2 mutation }\end{array}$ & $\begin{array}{l}\text { - Newly diagnosed acute } \\
\text { myeloid leukemia } \\
\text { - Untreated AML } \\
\text { - AML arising from } \\
\text { myelodysplastic syndrome } \\
\text { (MDS) } \\
\text { - AML arising from } \\
\text { antecedent hematologic } \\
\text { disorder } \\
\text { - AML arising after exposure } \\
\text { to genotoxic injury }\end{array}$ & $\begin{array}{l}\text { - Drug: AG-120 } \\
\text { - Drug: AG-22I } \\
\text { - Drug: Cytarabine } \\
\text { - Drug: Daunorubicin } \\
\text { - Drug: Idarubicin } \\
\text { - Drug: Mitoxantrone } \\
\text { - Drug: Etoposide }\end{array}$ & $153^{*}$ & Phase I \\
\hline NCT02074839 & $\begin{array}{l}\text { Study of orally administered } \\
\text { AG-I20 in subjects with } \\
\text { advanced hematologic } \\
\text { malignancies with an IDHI } \\
\text { mutation }\end{array}$ & $\begin{array}{l}\text { - Relapsed/Refractory acute } \\
\text { myeloid leukemia } \\
\text { - Untreated AML } \\
\text { - Other IDHI-mutated } \\
\text { Positive Hematologic } \\
\text { Malignancies } \\
\text { - Myelodysplastic Syndromes }\end{array}$ & - Drug: AG- 120 & 291 & Phase I \\
\hline
\end{tabular}

Notes: Data from www.clinicaltrials.gov (January 2021). *Actual enrollment.

blocking drug/cofactor binding and/or occurrence of $I D H 2$ mutations, whose incidence is similar to that of mutations in the RTK pathway. ${ }^{63}$

Furthermore, data presented in a recently published review and case series, point to mutant $I D H$ isoform switching, from cytoplasmic mutant $I D H 1$ to mitochondrial mutant $I D H 2$ or in the reverse order, as the primary mechanism responsible for acquired resistance to IDH inhibitors. ${ }^{27,64}$ 2-HG production in AML with mutated $I D H$ is suggested to be mediated by selective pressure. To circumvent drug resistance in this setting, consecutive administration of IDH inhibitors (either IDH1>IDH2 or IDH2>IDH1) is proposed. ${ }^{64}$

One of the ways to prevent the development of secondary resistance in AML is through the use of combination therapy. Several recent studies have demonstrated the synergistic effect of the combination of the BCL-2 inhibitor, venetoclax, either with hypomethylation agents or with low-dose cytarabine. ${ }^{65-67}$ The most responsive patients to both combination treatments were found to be those with IDH1/IDH2 and NPM1 mutations.

Based on the established efficacy of both IDH inhibitors and venetoclax in AMLs with mutant IDH1, potential synergistic action of their combinations is hypothesized. This issue is being currently investigated in a Phase Ib/II clinical trial assessing the use of venetoclax and ivosidenib with/without azacytidine in mutant IDHI R/R AML patients (NCT 03471260) (Table 1).

Preliminary results demonstrated an ORR of $75 \%$ with no significant added toxicity, suggesting a favorable risk/ benefit profile of these triplet combinations. ${ }^{68}$ These findings are promising, although Chan et al suggest an antagonist effect of the mentioned combination in human AML blast cells. ${ }^{69}$

Another promising combination therapy that might provide a synergistic effect could comprise mutant IDH 
inhibitors and RTK pathway inhibitors (including FLT3 inhibitors). Along the same lines, the currently investigated joint effect of mutant IDH1 and mutant IDH2 inhibition may also have a therapeutic potential in this patient population. $^{70}$
Among other open issues related to AML management are the safety and efficacy of combinations including novel agents only or those incorporating standard chemotherapy for AML. Prospective clinical studies are warranted to address these questions.

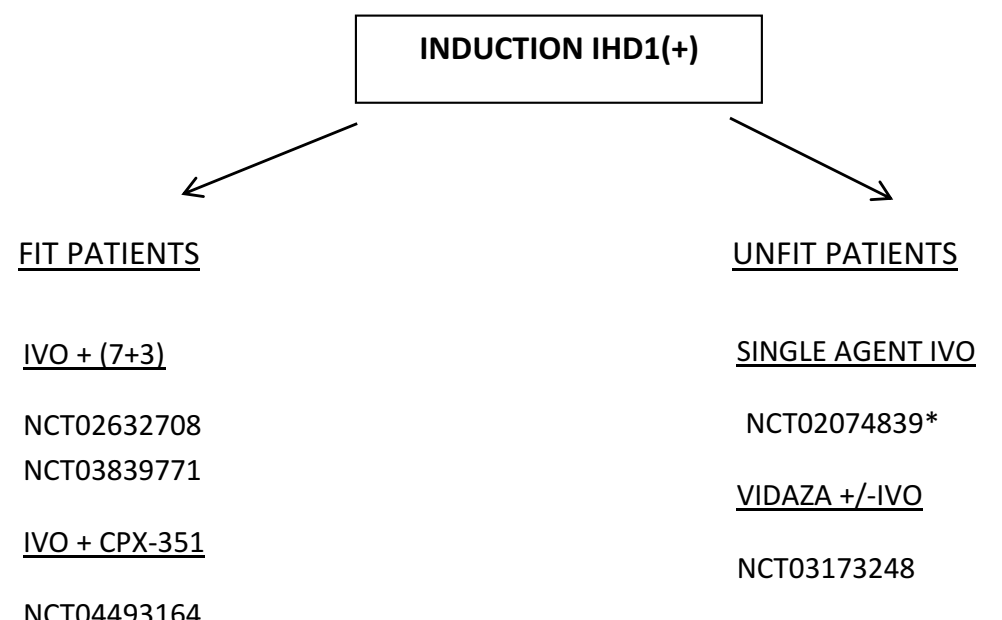

$\underline{\text { IVO+VENETOCLAX +/- VIDAZA }}$

NCT03471260

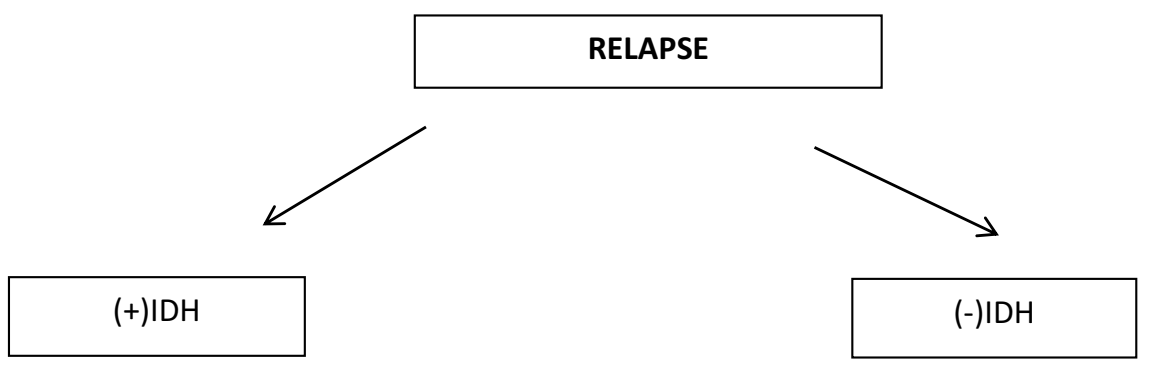

SINGLE AGENT IVO

SALVAGE COMBINATIONS

NCT02074839*

NCT03245424**

$\underline{\text { IVO+ COMB. CHEMO }}$

NCT04250051

* approval study

**post marketing

Figure I How to incorporate ivosidenib in AML treatment. 


\section{Summary and Conclusions}

The general therapeutic strategy in patients with AML has significantly changed in the last years. Although younger patients are still mainly treated with the combination of an anthracycline and cytosine-arabinoside, as induction therapy, the growing knowledge of molecular and genetic landscape of AML has led to novel treatment options, for both younger and older patients. ${ }^{71-74} I D H 1$ mutations occur in up of $20 \%$ patients with AML. ${ }^{4,18,19}$

The mutated IDH1 enzyme causes the formation of $2 \mathrm{HG}$ instead of $\alpha \mathrm{KG}$, which entails a decrease in $\alpha \mathrm{KG}$ levels with an increase in 2HG. The latter metabolite functions as a competitive inhibitor of $\alpha \mathrm{KG}$-dependent reactions and causes hypermethylation of DNA and histones resulting in differentiation block. It is postulated that this sequence of events promotes leukemogenesis.

The availability of a large number of novel drugs for the treatment of newly diagnosed AML makes it possible to explore new treatment combinations. Yet, choosing the optimal drug combination and schedule of its administration, not associated with excessive toxicity, is challenging.

Ivosidenib is the first IDH mutated inhibitor that was approved by FDA for R/R mutated IDH AML patients, based on results from NCT02074839 study and was administered as monotherapy. ${ }^{50}$

Other randomized phase I/II trials are currently being conducted to evaluate the efficacy and safety of ivosidenib as monotherapy or in combinations in this group of patients (NCT04250051, NCT04176393) (Table 1).

Several clinical trials are studying the combined effect of ivosidenib with intensive chemotherapy (NCT04493164, NCT02632708), hypomethylating agents (NCT03173248) or with other targeted therapies (NCT 0341260) in newly diagnosed IDHI-mutated AML patients.

Results from these trials will provide the necessary data whether to incorporate ivosidenib in the first-line treatment as standard of care for patients with IDHImutated AML. It is reasonable to assume that the addition of ivosidenib can lead to improvement in long-term survival. $^{2,37,61,68,75}$

Maintenance treatment is also being studied in several trials. The optimal duration of maintenance treatment and how effective it will be, is yet to be evaluated. Data on IDH1 mutation clearance and MRD might be of help to resolve this question, as was demonstrated by Chifotides et $\mathrm{al}^{37}$ and other studies.
A correlation between the MRD status and the cumulative risk for relapse in AML patients was demonstrated by several studies. The long-term clinical impact of $I D H$ mutation clearance is under investigation. Preliminary data suggest that clearance of the mutation results in a prolonged duration of response and improved overall survival. ${ }^{5,37,61}$

With ivosidenib, adverse events of special interest are the IDH-DS, leukocytosis and prolongation of the QT interval, which can be managed with appropriate guidance.

The drug is well tolerated and the majority of treated patients achieve composite complete remission (hematologic improvement, remission without hematologic improvement and complete remission). This finding correlates with a prolongation in overall survival - as was demonstrated by the above mentioned studies.

Figure 1 summarizes the role of Ivosidenib in the treatment of AML.

Resistance to ivosidenib ${ }^{50,63}$ may lead to disease progression with increased 2-HG concentration in plasma and re-emergence of leukemic blasts. This emphasizes the need for therapeutic approaches enabling prevention of drug resistance.

In conclusion, ivosidenib is a promising novel agent for the treatment of IDH1-mutated AML in the era of targeted therapy for AML. Yet, there is still a need for prospective double-blind studies to confirm its role and timing in the treatment of AML.

\section{Acknowledgments}

We wish to thank Sonia Kamenetsky for expert assistance in the preparation of this manuscript.

\section{Author Contributions}

All authors made a significant contribution to the work reported, whether that is in the conception, study design, execution, acquisition of data, analysis and interpretation, or in all these areas; took part in drafting, revising or critically reviewing the article; gave final approval of the version to be published; have agreed on the journal to which the article has been submitted; and agree to be accountable for all aspects of the work.

\section{Funding}

There is no funding to report.

\section{Disclosure}

The authors declare no potential conflicts of interest. 


\section{References}

1. Döhner H, Estey E, Grimwade D, et al. Diagnosis and management of AML in adults: 2017 ELN recommendations from an international expert panel. Blood. 2017;129(4):424-447.

2. DiNardo CD, Jabbour E, Ravandi F, et al. IDH1 and IDH2 mutations in myelodysplastic syndromes and role in disease progression. Leukemia. 2016;30(4):980-984. doi:10.1038/leu.2015. 211

3. Rowe JM. Progress and predictions: AML in 2018. Best Pract Res Clin Haematol. 2018;31(4):337-340. doi:10.1016/j.beha.2018.09. 002

4. Mardis ER, Ding L, Dooling DJ, et al. Recurring mutations found by sequencing an acute myeloid leukemia genome. $N$ Engl $\mathrm{J}$ Med. 2009;361(11):1058-1066. doi:10.1056/NEJMoa0903840

5. Donker ML, Ossenkoppele GJ. Evaluating ivosidenib for the treatment of acute myeloid leukemia. Expert Opin Pharmacother. 2020;1-9.

6. Warburg O. On the origin of cancer cells. Science. 1956;123 (3191):309-314. doi:10.1126/science.123.3191.309

7. Vander Heiden MG, Cantley LC, Thompson CB. Understanding the Warburg effect: the metabolic requirements of cell proliferation. Science. 2009;324(5930):1029-1033. doi:10.1126/ science. 1160809

8. Molenaar RJ, Maciejewski JP, Wilmink JW, van Noorden CJF. Wildtype and mutated IDH1/2 enzymes and therapy responses. Oncogene. 2018;37(15):1949-1960. doi:10.1038/s41388-017-0077-z

9. Avellaneda Matteo D, Grunseth AJ, Gonzalez ER, et al. Molecular mechanisms of isocitrate dehydrogenase 1 (IDH1) mutations identified in tumors: the role of size and hydrophobicity at residue 132 on catalytic efficiency. J Biol Chem. 2017;292(19):7971-7983. doi:10.1074/jbc.M117.776179

10. Losman J-A, Looper RE, Koivunen P, et al. (R)-2-hydroxyglutarate is sufficient to promote leukemogenesis and its effects are reversible. Science. 2013;339(6127):1621-1625. doi:10.1126/science.1231677

11. McCarthy N. Leukaemia: knowing left from right. Nat Rev Cancer. 2013;13(4):220-221. doi:10.1038/nrc3487

12. Popovici-Muller J, Lemieux RM, Artin E, et al. Discovery of AG-120 (Ivosidenib): a first-in-class mutant IDH1 inhibitor for the treatment of IDH1 mutant cancers. ACS Med Chem Lett. 2018;9(4):300-305. doi:10.1021/acsmedchemlett.7b00421

13. Parsons DW, Jones S, Zhang X, et al. An integrated genomic analysis of human glioblastoma multiforme. Science. 2008;321(5897): 1807-1812. doi:10.1126/science. 1164382

14. Losman J-A, Kaelin WG. What a difference a hydroxyl makes: mutant IDH, (R)-2-hydroxyglutarate, and cancer. Genes Dev. 2013;27(8):836-852. doi:10.1101/gad.217406.113

15. Yan H, Parsons DW, Jin G, et al. IDH1 and IDH2 mutations in gliomas. $N$ Engl $J$ Med. 2009;360(8):765-773. doi:10.1056/ NEJMoa0808710

16. Boscoe AN, Rolland C, Kelley RK. Frequency and prognostic significance of isocitrate dehydrogenase 1 mutations in cholangiocarcinoma: a systematic literature review. $J$ Gastrointest Oncol. 2019;10 (4):751-765. doi:10.21037/jgo.2019.03.10

17. Green A, Beer P. Somatic mutations of IDH1 and IDH2 in the leukemic transformation of myeloproliferative neoplasms. $N$ Engl $J$ Med. 2010;362(4):369-370. doi:10.1056/NEJMc0910063

18. Schnittger S, Haferlach C, Ulke M, Alpermann T, Kern W, Haferlach T. IDH1 mutations are detected in $6.6 \%$ of 1414 AML patients and are associated with intermediate risk karyotype and unfavorable prognosis in adults younger than 60 years and unmutated NPM1 status. Blood. 2010;116(25):5486-5496. doi:10.1182/blood2010-02-267955

19. Papaemmanuil E, Gerstung M, Bullinger L, et al. Genomic classification and prognosis in acute myeloid leukemia. $N$ Engl $\mathrm{J} \mathrm{Med.}$ 2016;374(23):2209-2221. doi:10.1056/NEJMoa1516192
20. Kao H-W, Liang DC, Kuo M-C, et al. High frequency of additional gene mutations in acute myeloid leukemia with MLL partial tandem duplication: DNMT3A mutation is associated with poor prognosis. Oncotarget. 2015;6(32):33217-33225. doi:10.18632/oncotarget.5202

21. Nassereddine S, Lap CJ, Tabbara IA. Evaluating ivosidenib for the treatment of relapsed/refractory AML: design, development, and place in therapy. Onco Targets Ther. 2019;12:303-308. doi:10.2147/ OTT.S182443

22. Kosmider O, Gelsi-Boyer V, Slama L, et al. Mutations of IDH1 and IDH2 genes in early and accelerated phases of myelodysplastic syndromes and MDS/myeloproliferative neoplasms. Leukemia. 2010;24(5):1094-1096. doi:10.1038/leu.2010.52

23. Desai P, Mencia-Trinchant N, Savenkov O, et al. Somatic mutations precede acute myeloid leukemia years before diagnosis. Nat Med. 2018;24(7):1015-1023. doi:10.1038/s41591-018-0081-z

24. Luque Paz D, Jouanneau-Courville R, Riou J, et al. Leukemic evolution of polycythemia vera and essential thrombocythemia: genomic profiles predict time to transformation. Blood Adv. 2020;4 (19):4887-4897. doi:10.1182/bloodadvances.2020002271

25. Haferlach T, Nagata Y, Grossmann V, et al. Landscape of genetic lesions in 944 patients with myelodysplastic syndromes. Leukemia. 2014;28(2):241-247. doi:10.1038/leu.2013.336

26. Molenaar RJ, Thota S, Nagata Y, et al. Clinical and biological implications of ancestral and non-ancestral IDH1 and IDH2 mutations in myeloid neoplasms. Leukemia. 2015;29(11):2134-2142. doi:10.1038/leu.2015.91

27. Testa U, Castelli G, Pelosi E. Isocitrate dehydrogenase mutations in myelodysplastic syndromes and in acute myeloid leukemias. Cancers (Basel). 2020;12(9). doi:10.3390/cancers12092427

28. Makishima H, Yoshizato T, Yoshida K, et al. Dynamics of clonal evolution in myelodysplastic syndromes. Nat Genet. 2017;49 (2):204-212. doi:10.1038/ng.3742

29. Patnaik MM, Hanson CA, Hodnefield JM, et al. Differential prognostic effect of IDH1 versus IDH2 mutations in myelodysplastic syndromes: a Mayo Clinic Study of 277 patients. Leukemia. 2012;26(1):101-105. doi:10.1038/leu.2011.298

30. Wang N, Wang F, Shan N, Sui X, Xu H. IDH1 mutation is an independent inferior prognostic indicator for patients with myelodysplastic syndromes. Acta Haematol. 2017;138(3):143-151. doi:10. $1159 / 000479546$

31. Bruneau J, Molina TJ. WHO classification of tumors of hematopoietic and lymphoid tissues. In: van Krieken JHJM, editor. Encyclopedia of Pathology. Cham: Springer International Publishing; 2019:1-5.

32. Tefferi A, Lasho TL, Finke CM, et al. Targeted deep sequencing in primary myelofibrosis. Blood Adv. 2016;1(2):105-111. doi:10.1182/ bloodadvances. 2016000208

33. Pardanani A, Lasho TL, Finke CM, Mai M, McClure RF, Tefferi A. IDH1 and IDH2 mutation analysis in chronic- and blast-phase myeloproliferative neoplasms. Leukemia. 2010;24(6):1146-1151. doi:10. 1038/leu.2010.77

34. Bartels S, Faisal M, Büsche G, et al. Mutations associated with age-related clonal hematopoiesis in PMF patients with rapid progression to myelofibrosis. Leukemia. 2020;34(5):1364-1372. doi:10.10 38/s41375-019-0668-5

35. Lasho TL, Jimma T, Finke CM, et al. SRSF2 mutations in primary myelofibrosis: significant clustering with IDH mutations and independent association with inferior overall and leukemia-free survival. Blood. 2012;120(20):4168-4171. doi:10.1182/blood-2012-05-429696

36. Tefferi A, Jimma T, Sulai NH, et al. IDH mutations in primary myelofibrosis predict leukemic transformation and shortened survival: clinical evidence for leukemogenic collaboration with JAK2V617F. Leukemia. 2012;26(3):475-480. doi:10.1038/leu.2011.253

37. Chifotides HT, Masarova L, Alfayez M, et al. Outcome of patients with IDH1/2-mutated post-myeloproliferative neoplasm AML in the era of IDH inhibitors. Blood Adv. 2020;4(21):5336-5342. doi:10. 1182/bloodadvances.2020001528 
38. Paschka P, Schlenk RF, Gaidzik VI, et al. IDH1 and IDH2 mutations are frequent genetic alterations in acute myeloid leukemia and confer adverse prognosis in cytogenetically normal acute myeloid leukemia with NPM1 mutation without FLT3 internal tandem duplication. $J$ Clin Oncol. 2010;28(22):3636-3643. doi:10.1200/JCO.2010.28.3762

39. Patel JP, Gönen M, Figueroa ME, et al. Prognostic relevance of integrated genetic profiling in acute myeloid leukemia. $N$ Engl J Med. 2012;366(12):1079-1089. doi:10.1056/NEJMoa1112304

40. Falini B, Spinelli O, Meggendorfer M, et al. IDH1-R132 changes vary according to NPM1 and other mutations status in AML. Leukemia. 2019;33(4):1043-1047. doi:10.1038/s41375-018-0299-2

41. Figueroa ME, Abdel-Wahab O, Lu C, et al. Leukemic IDH1 and IDH2 mutations result in a hypermethylation phenotype, disrupt TET2 function, and impair hematopoietic differentiation. Cancer Cell. 2010;18(6):553-567. doi:10.1016/j.ccr.2010.11.015

42. Green CL, Evans CM, Hills RK, Burnett AK, Linch DC, Gale RE. The prognostic significance of IDH1 mutations in younger adult patients with acute myeloid leukemia is dependent on FLT3/ITD status. Blood. 2010;116(15):2779-2782. doi:10.1182/blood-2010-02-270926

43. DiNardo CD, Ravandi F, Agresta S, et al. Characteristics, clinical outcome, and prognostic significance of IDH mutations in AML. $\mathrm{Am}$ $J$ Hematol. 2015;90(8):732-736. doi:10.1002/ajh.24072

44. Xu Q, Li Y, Lv N, et al. Correlation between isocitrate dehydrogenase gene aberrations and prognosis of patients with acute myeloid leukemia: a systematic review and meta-analysis. Clin Cancer Res. 2017;23(15):4511-4522. doi:10.1158/1078-0432.CCR-16-2628

45. Dunlap JB, Leonard J, Rosenberg M, et al. The combination of NPM1, DNMT3A, and IDH1/2 mutations leads to inferior overall survival in AML. Am J Hematol. 2019;94(8):913-920. doi:10.1002/ ajh. 25517

46. Suijker J, Oosting J, Koornneef A, et al. Inhibition of mutant IDH1 decreases D-2-HG levels without affecting tumorigenic properties of chondrosarcoma cell lines. Oncotarget. 2015;6(14):12505-12519. doi:10.18632/oncotarget. 3723

47. Fan B, Mellinghoff IK, Wen PY, et al. Clinical pharmacokinetics and pharmacodynamics of ivosidenib, an oral, targeted inhibitor of mutant IDH1, in patients with advanced solid tumors. Invest New Drugs. 2020;38(2):433-444. doi:10.1007/s10637-019-00771-x

48. Dai D, Yang H, Nabhan S, et al. Effect of itraconazole, food, and ethnic origin on the pharmacokinetics of ivosidenib in healthy subjects. Eur J Clin Pharmacol. 2019;75(8):1099-1108. doi:10.10 07/s00228-019-02673-6

49. Megías-Vericat JE, Solana-Altabella A, Ballesta-López O, MartínezCuadrón D, Montesinos P. Drug-drug interactions of newly approved small molecule inhibitors for acute myeloid leukemia. Ann Hematol. 2020;99(9):1989-2007. doi:10.1007/s00277-020-04186-0

50. DiNardo CD, Stein EM, de Botton S, et al. Durable remissions with ivosidenib in IDH1-mutated relapsed or refractory AML. $N$ Engl J Med. 2018;378(25):2386-2398. doi:10.1056/NEJMoa1716984

51. Norsworthy KJ, Luo L, Hsu V, et al. FDA approval summary: ivosidenib for relapsed or refractory acute myeloid leukemia with an isocitrate dehydrogenase-1 mutation. Clin Cancer Res. 2019;25 (11):3205-3209. doi:10.1158/1078-0432.CCR-18-3749

52. DiNardo CD, Stein EM. SOHO state of the art update and next questions: IDH therapeutic targeting in AML. Clin Lymphoma Myeloma Leuk. 2018;18(12):769-772. doi:10.1016/j.clml.2018.10.007

53. Maertens JA, Girmenia C, Brüggemann RJ, et al. European guidelines for primary antifungal prophylaxis in adult haematology patients: summary of the updated recommendations from the European Conference on Infections in Leukaemia. $J$ Antimicrob Chemother. 2018;73(12):3221-3230. doi:10.1093/jac/dky286

54. Safdar A, Rodriguez GH. Aerosolized amphotericin B lipid complex as adjunctive treatment for fungal lung infection in patients with cancer-related immunosuppression and recipients of hematopoietic stem cell transplantation. Pharmacotherapy. 2013;33(10):10 35-1043. doi:10.1002/phar.1309
55. Boutzen H, Saland E, Larrue C, et al. Isocitrate dehydrogenase 1 mutations prime the all-trans retinoic acid myeloid differentiation pathway in acute myeloid leukemia. J Exp Med. 2016;213 (4):483-497. doi:10.1084/jem.20150736

56. Birendra KC, DiNardo CD. Evidence for clinical differentiation and differentiation syndrome in patients with acute myeloid leukemia and IDH1 mutations treated with the targeted mutant IDH1 inhibitor, AG-120. Clin Lymphoma Myeloma Leuk. 2016;16(8):460-465. doi:10.1016/j.clml.2016.04.006

57. Hernandez Burgos P, Patel J, Chen A. Ivosidenib induction therapy complicated by myopericarditis and cardiogenic shock: a case report and literature review. J Oncol Pharm Pract. 2020;26(3):754-757. doi:10.1177/1078155219865934

58. Norsworthy KJ, Mulkey F, Scott EC, et al. Differentiation syndrome with ivosidenib and enasidenib treatment in patients with relapsed or refractory IDH-mutated AML: a U.S. food and drug administration systematic analysis. Clin Cancer Res. 2020;26(16):4280-4288. doi:10.1158/1078-0432.CCR-20-0834

59. Martelli MP, Martino G, Cardinali V, Falini B, Martinelli G, Cerchione C. Enasidenib and ivosidenib in AML. Minerva Med. 2020;111(5). doi:10.23736/S0026-4806.20.07024-X

60. Roboz GJ, DiNardo CD, Stein EM, et al. Ivosidenib induces deep durable remissions in patients with newly diagnosed IDH1-mutant acute myeloid leukemia. Blood. 2020;135(7):463-471. doi:10.1182/ blood.2019002140

61. Stein EM, DiNardo CD, Fathi AT, et al. Ivosidenib or enasidenib combined with intensive chemotherapy in patients with newly diagnosed AML: a phase 1 study. Blood. 2020;135 (7):463-471.

62. FDA approves ivosidenib as first-line treatment for AML with IDH1 mutation. FDA [Internet]. [cited August 31, 2020]. Available from: https://www.fda.gov/drugs/resources-information-approved-drugs /fda-approves-ivosidenib-first-line-treatment-aml-idh1-mutation. Accessed May 25, 2021.

63. Choe S, Wang H, DiNardo CD, et al. Molecular mechanisms mediating relapse following ivosidenib monotherapy in IDH1-mutant relapsed or refractory AML. Blood Adv. 2020;4(9):1894-1905. doi:10.1182/bloodadvances.2020001503

64. Harding JJ, Lowery MA, Shih AH, et al. Isoform switching as a mechanism of acquired resistance to mutant isocitrate dehydrogenase inhibition. Cancer Discov. 2018;8(12):1540-1547. doi:10.1158/ 2159-8290.CD-18-0877

65. DiNardo CD, Jonas BA, Pullarkat V, et al. Azacitidine and venetoclax in previously untreated acute myeloid leukemia. $N$ Engl J Med. 2020;383(7):617-629. doi:10.1056/ NEJMoa2012971

66. Tiong IS, Dillon R, Ivey A, et al. Venetoclax induces rapid elimination of NPM1 mutant measurable residual disease in combination with low-intensity chemotherapy in acute myeloid leukaemia. $\mathrm{Br}$ J Haematol. 2020;192(6):1026-1030. doi:10.1111/bjh.16722

67. Wei AH, Montesinos P, Ivanov V, et al. Venetoclax plus LDAC for newly diagnosed AML ineligible for intensive chemotherapy: a Phase 3 randomized placebo-controlled trial. Blood. 2020;135 (24):2137-2145. doi:10.1182/blood.2020004856

68. Dinardo C, Takahashi K, Kadia T, et al. Pf291 a phase 1b/2 clinical study of targeted idh1 inhibition with ivosidenib, in combination with the bcl-2 inhibitor venetoclax, for patients with idh1-mutated (midh1) myeloid malignancies. HemaSphere. 2019;3(S1):97. doi:10.1097/01. HS9.0000559376.17429.23

69. Chan SM, Thomas D, Corces-Zimmerman MR, et al. Isocitrate dehydrogenase 1 and 2 mutations induce BCL-2 dependence in acute myeloid leukemia. Nat Med. 2015;21(2):178-184. doi: $10.1038 / \mathrm{nm} .3788$

70. Ma R, Yun C-H. Crystal structures of pan-IDH inhibitor AG-881 in complex with mutant human IDH1 and IDH2. Biochem Biophys Res Commun. 2018;503(4):2912-2917. doi:10.1016/j.bbrc.2018.08.068 
71. Goldenson BH, Goodman AM, Ball ED, Goldenson BH, Goodman AM, Ball ED. Gemtuzumab ozogamicin for the treatment of acute myeloid leukemia in adults. Expert Opin Biol Ther. 2020;1-14. doi:10.1080/14712598.2021.1825678

72. Lambert J, Pautas C, Terré C, et al. Gemtuzumab ozogamicin for de novo acute myeloid leukemia: final efficacy and safety updates from the open-label, Phase III ALFA-0701 trial. Haematologica. 2019;104 (1):113-119. doi:10.3324/haematol.2018.188888

73. Stone RM, Mandrekar SJ, Sanford BL, et al. Midostaurin plus chemotherapy for acute myeloid leukemia with a FLT3 mutation. $N$ Engl J Med. 2017;377(5):454-464. doi:10.1056/NEJMoa1614359
74. DiNardo CD, Tiong IS, Quaglieri A, et al. Molecular patterns of response and treatment failure after frontline venetoclax combinations in older patients with AML. Blood. 2020;135(11):791-803. doi:10.1182/blood.2019003988

75. Lachowiez CA, Borthakur G, Loghavi S, et al. Phase Ib/II study of the IDH1-mutant inhibitor ivosidenib with the BCL2 inhibitor venetoclax \pm azacitidine in IDH1-mutated hematologic malignancies. JCO. 2020;38(15_suppl):7500. doi:10.1200/JCO.2020.38.15_suppl. 7500

\section{Publish your work in this journal}

Blood and Lymphatic Cancer: Targets and Therapy is an international, peer-reviewed, open access journal focusing on blood and lymphatic cancer research, identification of therapeutic targets and the optimal use of preventative and integrated treatment interventions to achieve improved outcomes, enhanced survival and quality of life for the cancer patient. The manuscript management system is completely online and includes a very quick and fair peer-review system. Visit http://www.dovepress.com/testimonials.php to read real quotes from published authors. 